Hier steht eine Anzeige.

Springer

\title{
Auflösung
}

\section{Erythroplasie Queyrat}

$\mathrm{D}$ ie histopathologische Untersuchung einer Hautbiopsie aus dem Herd ergab die Diagnose einer Erythroplasie Queyrat. Es handelt sich hierbei um ein Carcinoma in situ.

\section{Therapie und Verlauf}

Wegen des möglichen Überganges in ein spinozelluläres Karzinom wurde eine $\mathrm{CO}_{2}$-Lasertherapie durchgeführt. Postoperativ fanden regelmäßige Befundkontrollen statt.

Den klinischen Befund, bestehend aus einem über längere Zeit bestehenden, umschriebenen, geröteten Herd im Bereich der Glans penis und des inneren Präputialraumes, bezeichnet man als Erythroplakie.

\section{Differenzialdiagnosen}

Differenzialdiagnostisch ist in erster Linie an umschriebene chronische Balanoposthitis, Balanoposthitis circumscripta plasmacellularis (Zoon), Morbus Bowen, Psoriasis vulgaris, Lichen ruber, fixes Arzneimittelexanthem oder eben an Erythroplasie Queyrat zu denken. Bei der Diagnosestellung sind das Alter des Patienten, der Verlauf und der klinische
Befund zu berücksichtigen. Die Erythroplasie Queyrat und der Morbus Bowen kommen jenseits des fünften Lebensjahrzehntes als isolierte Herde vor.

Eine Psoriasis vulgaris ist besonders dann naheliegend, wenn weitere für Psoriasis typische Hautveränderungen bei dem Patienten nachweisbar sind. Das gleiche gilt auch für den Lichen ruber, wobei hier eine anuläre Konfiguration ein typisches klinisches Merkmal sein kann. Auch hier sind das Hautorgan und vor allem die Mundhöhle zu inspizieren, um die Erkrankung zu erkennen oder auszuschließen. Fixe Arzneimittelexantheme treten nach wiederholter Einnahme des auslösenden Medikaments immer an der gleichen Stelle auf und heilen mit einer bräunlichen residualen Pigmentierung ab. Im Zweifelsfall ist es sinnvoll, eine Hautbiopsie zu entnehmen, um die Diagnose histopathologisch zu sichern und ein Carcinoma in situ auszuschließen.

PD Dr. med. habil. Thomas Jansen Höntroper Str. 102 44869 Bochum

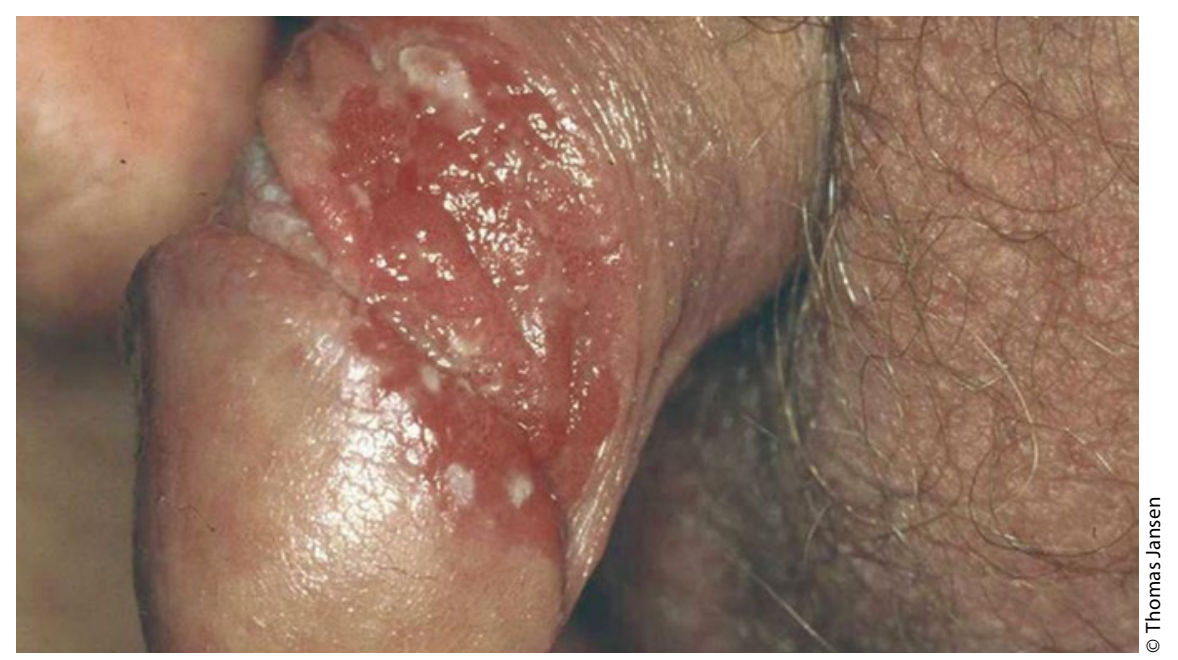

Erythroplasie Queyrat. 